\title{
Microarray-based analysis: Identification of hypoxia- regulated microRNAs in retinoblastoma cells
}

\author{
XIAOFANG XU ${ }^{1 *}$, RENBING JIA $^{1 *}$, YIXIONG ZHOU ${ }^{1}$, XIN SONG $^{1}$, JING WANG $^{1}$, \\ GUANXIANG QIAN $^{2}$, SHENGFANG GE ${ }^{1,2}$ and XIANQUN FAN ${ }^{1}$ \\ ${ }^{1}$ Department of Ophthalmology, Ninth People's Hospital, Shanghai Jiao Tong University School of Medicine, \\ 639 Zhi Zao Ju Road, Shanghai 200011; ²Department of Biochemistry and Molecular Biology, Shanghai Jiao \\ Tong University School of Medicine, 227 South Chong Qing Road, Shanghai 200025, P.R. China
}

Received November 23, 2010; Accepted February 2, 2011

DOI: 10.3892/ijo.2011.961

\begin{abstract}
Hypoxia is an essential feature of retinoblastoma and contributes to poor prognosis and resistance to conventional therapy. MicroRNAs (miRNAs) are small non-coding RNAs involved in a wide variety of biological processes, including cell differentiation, proliferation, death and metabolism. However, the relationship between hypoxia and the expression of miRNAs in retinoblastoma is not well understood. In this study, we aimed to analyze the pattern of miRNA expression in a retinoblastoma cell line under hypoxic conditions and to identify the miRNAs regulated by hypoxia, as well as their possible functions. miRNA expression profiling in retinoblastoma cells (HXO-RB44) under normal and hypoxic conditions was assessed by microarray techniques. The differentially expressed miRNAs were subjected to bioinformatic analyses to predict and categorise the key miRNAs and their target genes. A quantitative real-time RT-PCR approach was used to validate their expression. A Cell Counting kit was used to evaluate the functional significance of miR-181b in RB cell proliferation. There were 46 miRNAs that changed expression more than 2 -fold in response to hypoxia (34 up-regulated and 12 down-regulated). We identified a cluster of miRNAs that includes miR-181b, miR-125a-3p, miR-30c-2, miR-497 and miR-491-3p as hypoxiaregulated miRNAs (HRMs) in retinoblastoma cells, of which miR-181b was the most typically differentially expressed miRNA under hypoxic conditions. Functionally, these HRMs are involved in apoptosis, cell adhesion, cell proliferation and mRNA processing, all processes that associate closely with the hypoxia response of cancer cells. Additionally, we found that
\end{abstract}

Correspondence to: Professor Xianqun Fan, Department of Ophthalmology, Ninth People's Hospital, Shanghai Jiao Tong University School of Medicine, 639 Zhi Zao Ju Road, Shanghai 200011, P.R. China

E-mail: fanxq@sh163.net

${ }^{*}$ Contributed equally

Key words: microRNA, hypoxia, retinoblastoma, microarray analysis administration of miR-181b inhibitor can suppress proliferation of retinoblastoma cells. These findings provide the first evidence that miRNAs play an important role in the hypoxia response of retinoblastoma cells. MiR-181b, the most typically up-regulated miRNA may aid in future clinical intervention of retinoblastoma.

\section{Introduction}

Retinoblastoma is the most frequent intraocular cancer of children in China (1). In developed countries, over $95 \%$ of patients with retinoblastoma survive their malignancy (2). However, the prognosis for advanced cases is quite poor and metastasis to distant organs remains the leading cause of death in retinoblastoma cases. Hypoxia is one of the prime stress conditions present in the neoplastic microenvironment, hypoxic regions have been observed in retinoblastomas larger than $3.28 \mathrm{~mm}$ in LHBETATAG mice. Hypoxia can induce the expression of hypoxia-inducible factor (HIF), vascular endothelial growth factor (VEGF), matrix metalloproteases (MMPs) and cyclooxygenase-2 (COX-2) which are involved in cell adaptation to low oxygen in retinoblastoma and other cancers and contribute to poor prognosis or metastasis (3-7). Therefore, hypoxia may be a novel therapeutic target for treatment of advanced retinoblastoma (8).

MicroRNAs (miRNAs) are small non-coding RNAs (about 22 nucleotides in length) that bind to target mRNAs and prevent their translation into proteins (9). MiRNAs play critical roles in the coordination of a wide variety of processes including cell differentiation, proliferation, death and metabolism $(10,11)$. It is becoming clear that miRNAs correlates with the pathogenesis of cancers, influences the response of cancers to ionizing-radiation and chemotherapy $(12,13)$. Several reports have indicated that hypoxia influenced miRNAs expression profiles in a variety of cancer cells. Furthermore, a subgroup of these hypoxia-regulated microRNAs (termed HRMs) is believed to be necessary for survival of cancer cells in a low-oxygen environment. However, the relationship between hypoxia and the expression of miRNAs in retinoblastoma is not well clarified. Identification of cellular miRNAs that are regulated by hypoxia would provide valuable insight into tumor development as they may play a role in the hypoxia 
response of retinoblastoma. In the present study, we investigated HRMs in a human retinoblastoma cell line and analyzed their functions through miRNA microarray technology and computational analyses.

\section{Materials and methods}

Cell culture and growth conditions. Human retinoblastoma cell line HXO-RB44 cells (14) (a kind gift from Professor Xu Heping, Zhongnan University, China) were cultured in RPMI-1640 medium (Gibco, New York, NY) supplemented with $10 \%$ fetal bovine serum (FBS; Gibco). Hypoxic conditions were maintained in an InVivo200 hypoxia workstation (Biotrace International, Ruskinn Life Sciences, UK) with a steady flow of a low-oxygen gas mixture $\left(1 \% \mathrm{O}_{2}, 5 \% \mathrm{CO}_{2}, 94 \% \mathrm{~N}_{2}\right)$. Normoxic controls were propagated at $37^{\circ} \mathrm{C}$ and $5 \% \mathrm{CO}_{2}$. $\mathrm{HXO}-\mathrm{RB} 44$ cells in the exponential phase of growth were plated in 6 -well plates $\left(5 \times 10^{6}\right.$ cells per well) under hypoxic and normal conditions and incubated for $48 \mathrm{~h}$.

MicroRNA microarray assay. Total RNA was extracted using the TRIzol reagent (Invitrogen, Carlsbad, CA, USA) according to the manufacturer's protocol. RNA was labeled and hybridized to microRNA microarray chips as follows: $5 \mu \mathrm{g}$ of RNA from each sample was fluorescently labeled with the Hy3 or Hy5 dyes using miRCURY LNA ${ }^{\mathrm{TM}}$ Array Power Labeling kit (Cat no. 208032-A, Exiqon, Denmark). The kit provided a simple and fast 2-step protocol to label all miRNAs, including those from animals, plants and viruses. The labeled RNA molecules were then hybridized to the miRCURY LNA ${ }^{\mathrm{TM}}$ pre-spotted microarrays using the miRCURY LNA ${ }^{\mathrm{TM}}$ Array microarray kit (v.11.0-human,mouse \& rat, Exiqon, Denmark). The miRCURY LNA $^{\mathrm{TM}}$ microRNAArrays consisted of control probes, mismatch probes, and 1769 capture probes complementary to human, mouse, rat and their related viral sequences from the v.11.0 release of miRBase. The array also contained a number of proprietary human miRPlus ${ }^{\mathrm{TM}}$ sequences that are not yet in miRBase. All probes on the chip were quadruple spotted. After hybridization, signals were detected with a Genepix 4000B scanner (Molecular Devices, Downingtown, PA, USA). Images were quantified by a Genepix Pro 6.0 apparatus (Molecular Devices).

Analysis of microarray data. The raw data were normalized and analyzed. For each spot, the signal was corrected by subtracting background intensity (B) from foreground intensity $(F)$ on the chip, yielding a verified signal value $=\mathrm{F}-\mathrm{B}$. Those spots with verified signal values $<50$, as well as the control spots, were filtered. Median normalization was performed on the remaining spots to average of the results of the four spots for each microRNA. An n-fold-change tool was used to identify altered expression of specific microRNAs.

Gene ontology $(\mathrm{GO})$ analysis. $\mathrm{GO}$ analysis was performed to analyze the main functions of the putative target genes of the differentially expressed miRNAs based on the Gene Ontology database (http://www.geneontology.org/) (15). Fisher's exact test and the $\chi^{2}$ test were used to classify the GO categories, and the false discovery rate (FDR) was used to correct the p-values. Enrichment provided a measure of the significance of the function: as the enrichment increases, the corresponding function is more specific. This approach aids in identifying those GO categories with more concrete functional descriptions that are represented in the experiment. Within the significant category ( $<<0.0005$ and FDR $<0.005$ as a threshold), the enrichment $\operatorname{Re}$ was calculated using the equation: $\operatorname{Re}=(\mathrm{nf} / \mathrm{n}) /(\mathrm{Nf} / \mathrm{N})$ where $n f$ is the number of different genes within the particular category, $\mathrm{n}$ is the total number of genes within the category, and $\mathrm{Nf}$ and $\mathrm{N}$ represent the number of differentially expressed genes and total genes in the entire microarray, respectively.

MicroRNA-Gene-Network analysis. The relationship between the differentially expressed miRNAs and target genes were determined according to the interactions between miRNAs and genes in the Sanger MicroRNA database (http://microrna. sanger. ac.uk/targets/v5/) using the Matlab software (MathWorks, Inc., USA). The microRNA-Gene-Network was built by comparing the genes expressed especially in the eye, identified using the NCBI unigene database (http://www.ncbi.nlm.nih. gov/unigene) on the basis of tissue specificity, to the differentially expressed miRNAs. The center of the network was represented by degree, which is defined as the contribution of one miRNA to those genes around it.

MicroRNA-GO-Network analysis. The microRNA-GO-Network was built according to the relationships between significant GO categories and genes and the relationships between microRNAs and genes. The center of this network was also represented by degree, revealing the control of miRNAs on GO categories.

TaqMan quantitative real-time PCR of miRNAs. The miRNAs with high degree in the network were selected for verification by reverse transcript and quantitative PCR. The RT and PCR reactions were performed using the Hairpin-it ${ }^{\mathrm{TM}}$ miRNAs Real-Time PCR Quantitation Kit (GenePharma Co. Ltd., Shanghai, China) according to the manufacturer's protocol. We used $2 \mu \mathrm{g}$ of total RNA in a $20-\mu 1$ reaction for reverse transcript reaction, the reaction was carried out at the following conditions: $30 \mathrm{~min}$ at $16^{\circ} \mathrm{C}, 30 \mathrm{~min}$ at $42^{\circ} \mathrm{C}, 5 \mathrm{~min}$ at $85^{\circ} \mathrm{C}$, and then held on $4^{\circ} \mathrm{C}$. The real-time PCR reaction was conducted in a final reaction volume of $25 \mu \mathrm{l}$ containing $2 \mu \mathrm{l}$ of miRNA RT product, $1.0 \mu \mathrm{l}$ of miRNA specific Primer set $(10 \mu \mathrm{M}), 0.2 \mu \mathrm{l}$ Taq DNA poly-merase $(5 \mathrm{U} / \mu \mathrm{l})$ and $10 \mu \mathrm{l}$ of $2 \mathrm{X}$ Master Mix. The PCR amplification protocol was $95^{\circ} \mathrm{C}$ for $1 \mathrm{~min}$ followed by 40 cycles of $95^{\circ} \mathrm{C}$ for $15 \mathrm{sec}, 62^{\circ} \mathrm{C}$ for $15 \mathrm{sec}, 72^{\circ} \mathrm{C}$ for $30 \mathrm{sec}$, and a 30 -sec final extension at $37^{\circ} \mathrm{C}$. The primers used for Q-PCR amplification were shown in Table I. The relative amounts of miRNAs were normalized against the $5 \mathrm{~S}$ rRNA, the fold change was calculated using the $2^{-\Delta \Delta \mathrm{Ct}}$ method and all experiments were performed in triplicate for each sample on a Rotogene 3000 real-time PCR system (Corbett Robotics, Australia).

Transfection of HXO-RB44 cells with MiR-181b inhibitor. HXO-RB44 cells in exponential phase of growth were seeded in 96-well plates $\left(6 \times 10^{3} / 100 \mu \mathrm{l} /\right.$ per well). MiR-181b inhibitor and negative control (Ambion, Inc.) were transfected into the cells at a final oligonucleotide concentration of $3 \mathrm{pmol} / \mathrm{well}$ using Lipofectamine 2000 (Invitrogen) according to the manufacturer's protocol. The wells added same amount of transfection agents were set as blank. The medium was replaced 
Table I. Primer sequence used for Q-PCR amplification.

\begin{tabular}{|c|c|c|}
\hline Name & Accession no. & Primer sequence $\left(5^{\prime}-3^{\prime}\right)$ \\
\hline hsa-mir-181b & MIMAT0000270 & $\begin{array}{l}\text { F:GGGAACATTCATTGCTG } \\
\text { R:CAGTGCGTGTCGTGGAGT }\end{array}$ \\
\hline hsa-mir-181d & MIMAT0002821 & $\begin{array}{l}\text { F:AACATTCATTGTTGTCGGTGGG } \\
\text { R:CATGATCAGCTGGGCCAAGA }\end{array}$ \\
\hline hsa-mir-125a-3p & MIMAT0004602 & $\begin{array}{l}\text { F:TGAGGTTCTTGGGAGCCA } \\
\text { R:GCAGCACAGAATTAATACGACTCAC }\end{array}$ \\
\hline hsa-miR-30c-2 & MIMAT0004550 & $\begin{array}{l}\text { F:TGGGAGAAGGCTGTTTACTCTAAAA } \\
\text { R:CATGATCAGCTGGGCCAAGA }\end{array}$ \\
\hline hsa-miR-497 & MIMAT0002820 & $\begin{array}{l}\text { F:GCAGCACACTGTGGTTTGTAAAA } \\
\text { R:CATGATCAGCTGGGCCAAGA }\end{array}$ \\
\hline hsa-let-7e & MIMAT0000066 & $\begin{array}{l}\text { F:TGAGGTAGGAGGTTGTATAGTTAAAAAA } \\
\text { R:CATGATCAGCTGGGCCAAGA }\end{array}$ \\
\hline hsa-miR-647 & MIMAT0003317 & $\begin{array}{l}\text { F:TGGCTGCACTCACTTCCTTCAAA } \\
\text { R:GCAGCACAGAATTAATACGACTCAC }\end{array}$ \\
\hline hsa-miR-491-3p & MIMAT0004765 & $\begin{array}{l}\text { F:ATGCAAGATTCCCTTCTACAAAaа } \\
\text { R:CATGATCAGCTGGGCCAAGA }\end{array}$ \\
\hline $5 \mathrm{~S}$ & V00589 & $\begin{array}{l}\text { F:CCATACCACCCTGAACGC } \\
\text { R:GTATTCCCAGGCGGTCTC }\end{array}$ \\
\hline
\end{tabular}

$\mathrm{F}$, indicates forward strand and $\mathrm{R}$, indicates reverse strand. 5S was used as endogenous control.

by fresh culture solution $6 \mathrm{~h}$ later. Cells were incubated for 24 or $48 \mathrm{~h}$ in a hypoxia or normal workstation, respectively.

Cell proliferation assay. Cell viability was determined by a Cell Counting Kit (CCK-8, Dojindo Laboratories, Kumamoto, Japan). Added $10 \mu \mathrm{l}$ of the CCK- 8 solution to each well of the plates, then incubated the plates for $2 \mathrm{~h}$ in the incubator and measured the absorbance at $450 \mathrm{~nm}$ with a microplate reader. The rate of cell proliferation was calculated according to the following equation:

$$
\text { rate of cell proliferation }=O D_{(\text {test })} / O D_{(\text {blank })} x 100 \%
$$

Statistical analysis. All experiments were performed in triplicate, and the data were expressed as mean \pm SD (standard deviation), unless otherwise noted. The comparative CT method was applied in the quantitative real-time RT-PCR assay according to the $\Delta \Delta \mathrm{CT}$ method. All statistical analyses were performed using the SPSS11.0 software. Analysis of variance (ANOVA) was used to analyze the difference between means with significance accepted as $\mathrm{p}<0.01$.

\section{Results}

Differentially expressed microRNAs in the human retinoblastoma cell line HXO-RB44 under hypoxic conditions, as compared to normal conditions. To explore the potential involvement of miRNAs in hypoxia response in retinoblastoma cells, we used a microarray-based screening method to evaluate differential expression of miRNAs after $48 \mathrm{~h}$ of hypoxia in retinoblastoma cells. Analysis of the microarray data demonstrated that 46 miRNAs were differentially expressed by at least 2-fold under hypoxic conditions. Of these differentially expressed miRNAs, 34 were up-regulated and 12 were downregulated (Fig. 1).

Microarray-based bioinformatics analyses revealed key miRNAs and their functions regulated by hypoxia. To investigate the relationship between the differentially expressed miRNAs and their functions in the hypoxia response, we performed bioinformatics analyses. GO analysis revealed the primary functions of the putative target genes of the differentially expressed miRNAs. According to the threshold of significant 


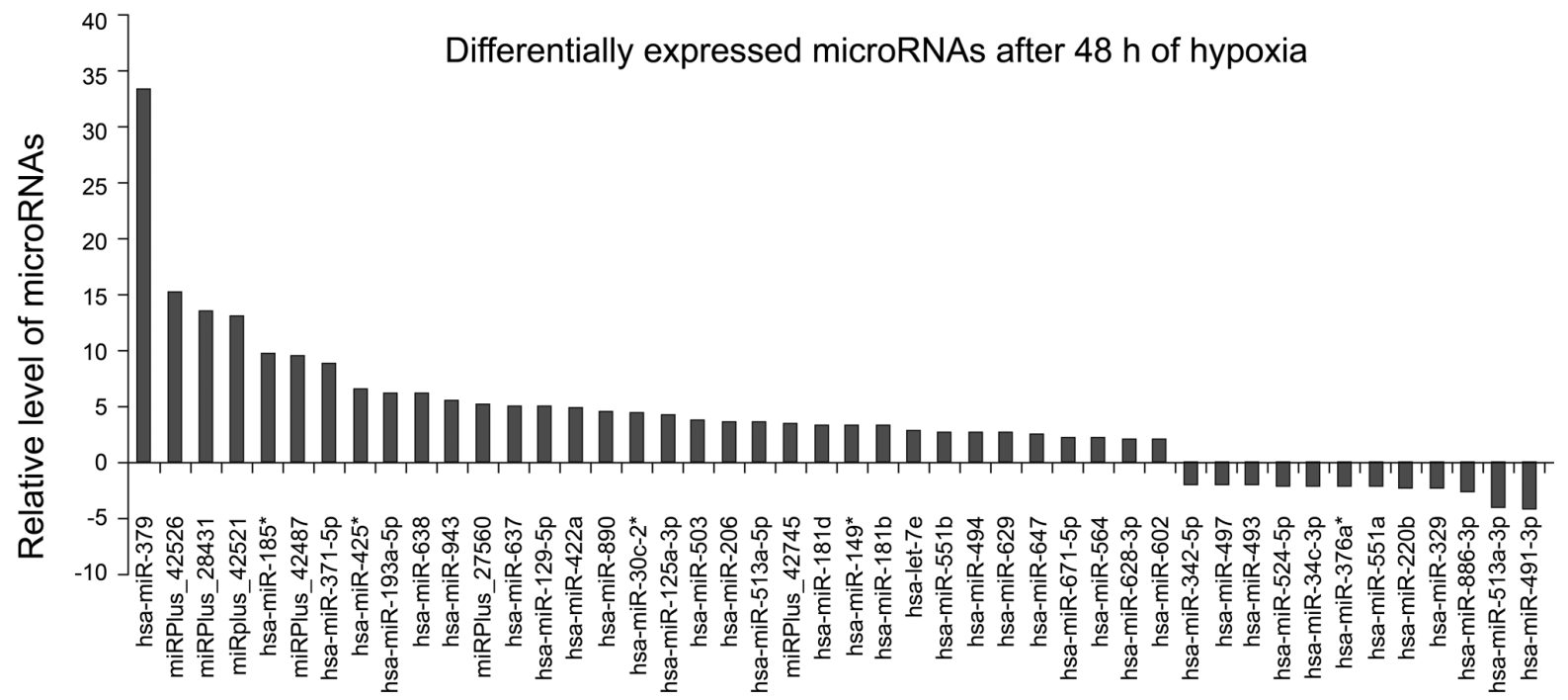

Figure 1. Differentially expressed miRNAs in human retinoblastoma cells (HXO-RB44) after $48 \mathrm{~h}$ of hypoxia, as compared to normal conditions. Different miRNAs are displayed on the $\mathrm{X}$ axis and the $\mathrm{Y}$ axis shows relative fold changes. The fold changes represent hypoxia versus normoxia.

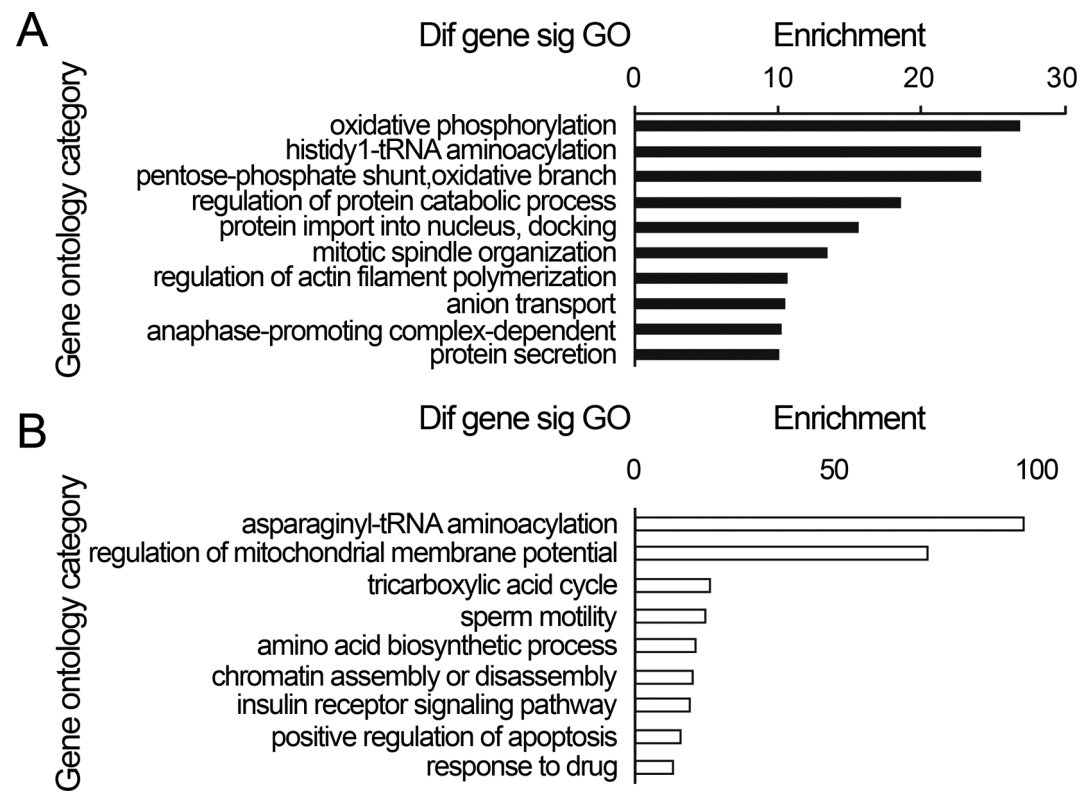

Figure 2. microRNAs target significant GO categories. (A) displays the GO categories targeted by up-regulated miRNAs; (B) displays the GO categories targeted by down-regulated miRNAs. The horizontal axis represents the enrichment of GO categories and the vertical axis represents the gene ontology categories.

GOs described in Materials and methods, the high-enrichment GO categories targeted by up-regulated miRNAs included oxidative phosphorylation, histidyl-tRNA aminoacylation, pentose-phosphate shunt and oxidative branch (see Fig. 2A for a complete list). Significant GO categories corresponding to down-regulated miRNAs included asparaginyl-tRNA aminoacylation, regulation of mitochondrial membrane potential and tricarboxylic acid cycle (see Fig. 2B for a complete list).

The microRNA-Gene-Networks (miRNA-mRNA regulatory network) generated based on the microarray results is shown in Fig. 3. Among the up-regulated miRNAs, miR-181b, 647, $30 \mathrm{c}-2$, 181d and 125a-3p were found to be key miRNAs with degrees of 62, 62, 61, 57 and 56, respectively (Fig. 3A). Of the down-regulated miRNAs, miR-34c-3p, 220b, 524-5p, 491-3p and 497 were determined to be key miRNAs with degrees of 27, 24, 22, 17 and 15 respectively (Fig. 3B). These findings suggested that these miRNAs might be pivotal for response to hypoxia. Thus, we considered these miRNAs as potential hypoxia-regulated miRNAs (HRMs) in retinoblastoma cells.

The microRNA-GO-Network analysis further revealed the functions of these HRMs. The major functions controlled by up-regulated HRMs were apoptosis, interspecies interaction between organisms, cell proliferation and cell adhesion with degrees of 27, 27, 26 and 25 respectively (Fig. 4A). The functions regulated by down-regulated HRMs were interspecies interaction between organisms, apoptosis and multicellular organismal development with degrees of 10,9 and 9, respectively (Fig. 4B). 


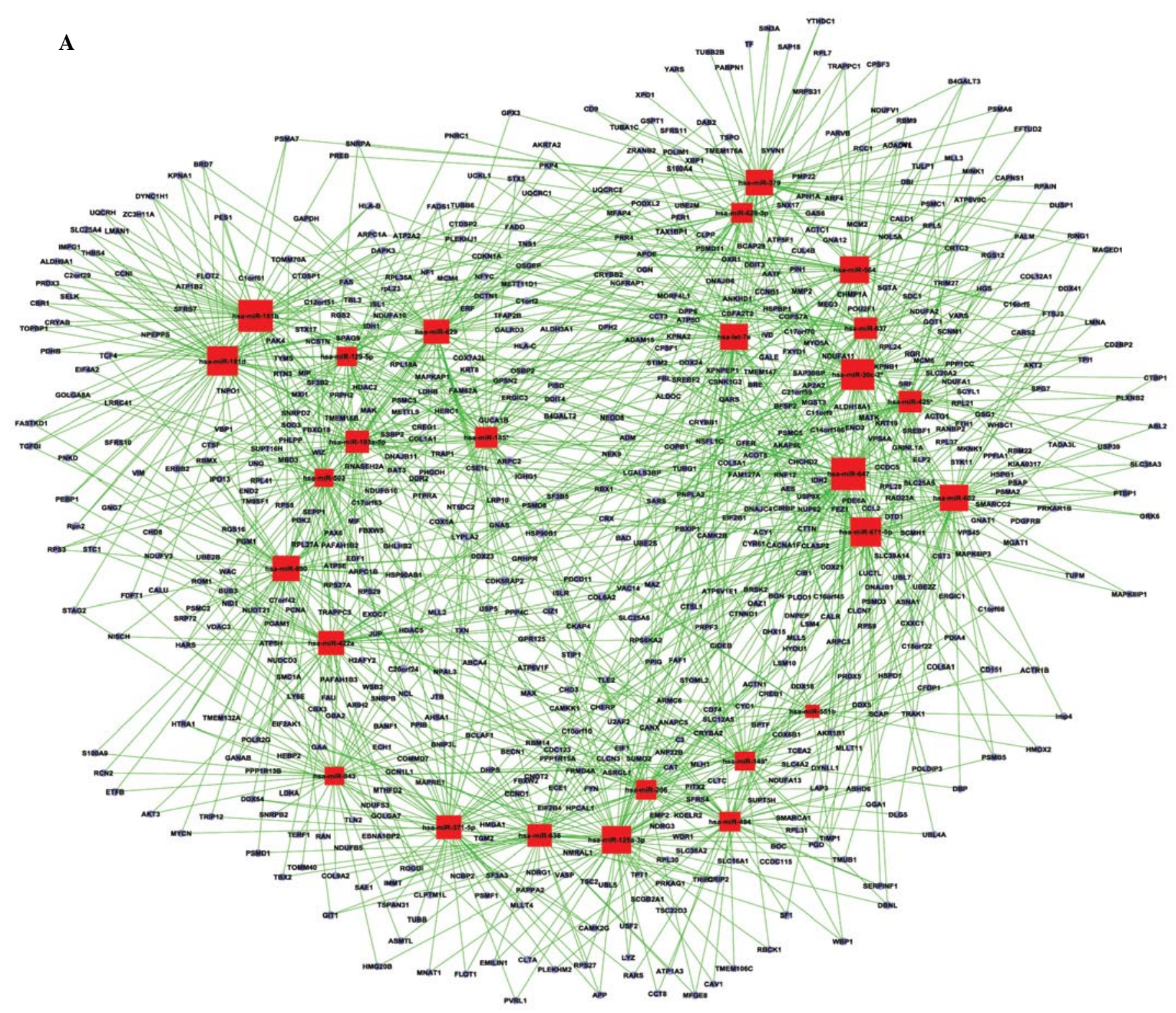

Figure 3. microRNA-gene-network. (A and B) show the microRNA-gene-networks of up-regulated and down-regulated miRNAs, respectively.

Detection of miRNA expression by quantitative real-time $R T$-PCR. To validate the microarray results, we chose eight potential HRMs (miR-181b, miR-125a-3p, let-7e, miR-497, miR-647, miR-30c-2, miR-181d and miR-491-3p) that displayed the highest degrees in the network for validation by quantitative real-time RT-PCR. Of these miRNAs, five were verified as significantly differentially expressed $(\mathrm{p}<0.01)$. The expression of miR-181b, miR-125a-3p and miR-30c-2 were up-regulated, while miR-497 and miR-491-3p were down-regulated, all of which are in agreement with the microarray results. However, the levels of miR-181d and let-7e showed no difference by PCR analysis while miR-647 showed the opposite expression pattern when compared to the microarray results (Fig. 5).

Effects of miR-181b inhibitor on the proliferation of HXO-RB44 cells. Since 'growth suppression' is one of the most important reactions of cells to hypoxia, we tested whether miR-181b, the most typically identified miRNA, can influence proliferation of RB cells. It showed that relative to control oligonucleotides, transfection of miR-181b inhibitor resulted in a significantly reduction of RB cells (Fig. 6).

\section{Discussion}

Hypoxia is a feature of retinoblastoma and may induce cellular hypoxia responses that alter the properties of cancer cells. While specific genes such as HIF and VEGF are induced by low oxygen, the transcription or translation of others is significantly suppressed. Recently, the study of gene repression in response to hypoxia has received increasing interest. One hypothesis states that miRNAs may be a regulatory factor in the response of cancer cells to hypoxia. Though the general mechanisms underlying gene regulation in response to hypoxia are not well understood, several studies have identified a group of miRNAs 


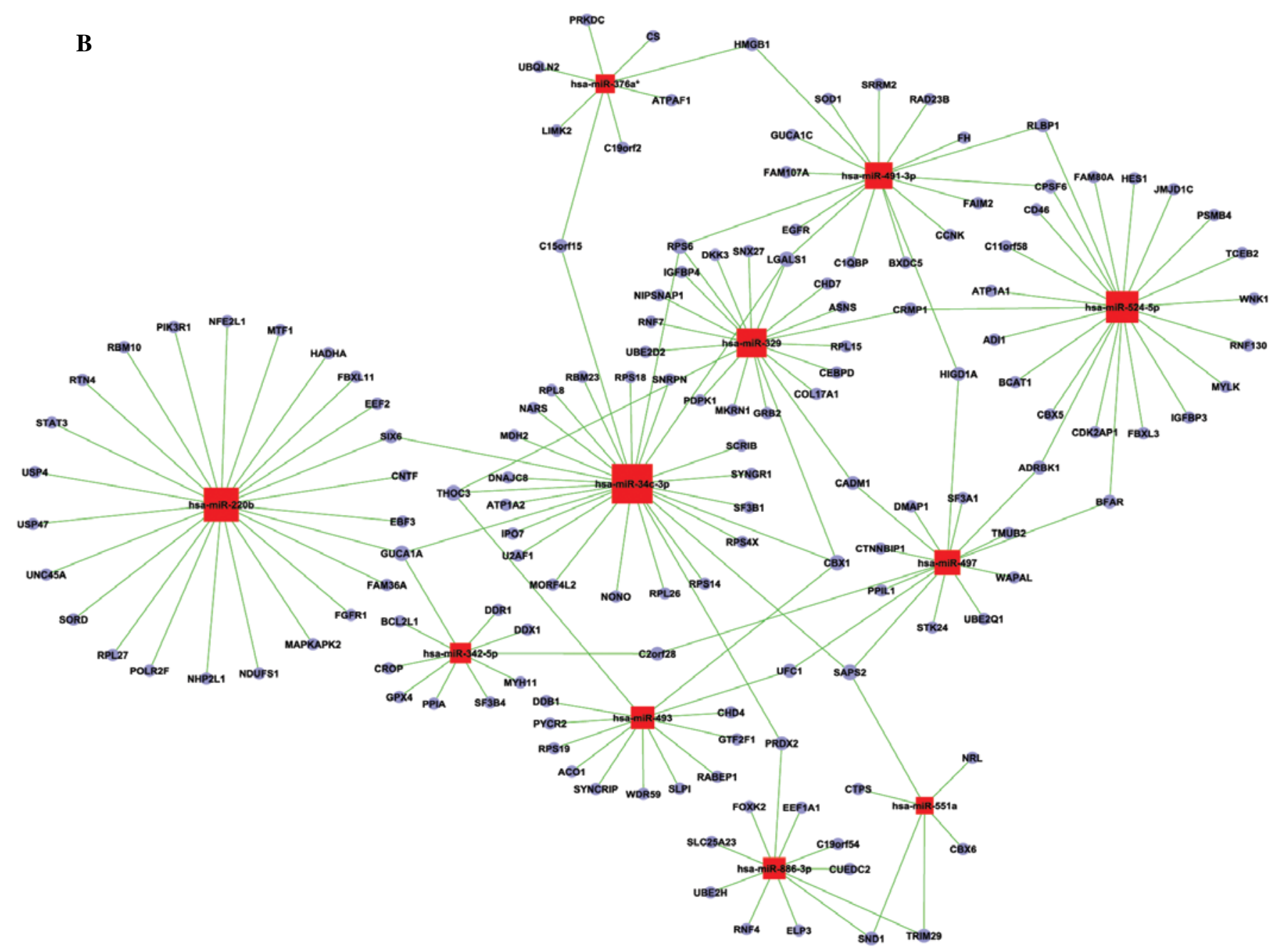

Figure 3 (continued). (A and B) show the microRNA-gene-networks of up-regulated and down-regulated miRNAs respectively. Ten miRNAs in total (miR181b, miR-647, miR-30c-2, miR-181d, miR-125a-3p, miR-34c-3p, miR-220b, miR-524-5p, miR-491-3p and miR-497) displayed highest degrees in the network and were considered as potential hypoxia-regulated miRNAs. Red square nodes represent miRNAs, light blue nodes represent genes and straight lines describe the regulatory relationship between miRNAs and genes.

(HRMs) that are closely associated with hypoxic environments. In silico studies have revealed a complex spectrum of candidate target genes of these HRMs including genes involving in cell proliferation, apoptosis, DNA repair, metabolism and migration. Thus, we speculate that HRMs may play a role in gene expression response to hypoxia with important consequences for cancer progression and response to therapy.

Our study provides the first profile of differential expression of miRNAs in retinoblastoma cells under hypoxic conditions. We identified 46 miRNAs that changed expression more than 2-fold in response to hypoxia. Through a microRNAGene-Network analysis, based on microarray technology that revealed accessory relationships between differentially expressed miRNAs and their target genes, we identified 10 miRNAs (miR-181b, miR-647, miR-30c-2, miR-181d, miR-125a-3p, miR-34c-3p, miR-220b, miR-524-5p, miR-491-3p and miR-497) as potential HRMs in retinoblastoma cells. Because of the potential biases of microarrays, we used quantitative real-time RT-PCR, which is a platform detection for mature and precursor miRNAs to validate the microarray data. The expression patterns of miR-181b, miR-125a-3p, miR-30c-2, miR-497 and $\mathrm{miR}-491-3 \mathrm{p}$ were found to agree with the results of the microarray. Compared to a variety of HRMs that have been identified in other cancers, including miR-210,21,23a, 23b, 24, 26a, 26b, 27a, 30b, 93, 103, 106a, 107, 125b, 181a,181b, 181c, 192, 195 and 213 (16), we find a limited overlap with our set of HRMs. However, these discrepancies were not necessarily surprising. Since miRNAs are often expressed in a tissuespecific and temporal manner, these differences are most likely related to the variability in the response to hypoxia of various cell-types, growth conditions (severity and duration of the hypoxic stress) or procedural aspects used in other studies (17-20). Our study provides a link between a tumor-specific stress factor (hypoxia) and gene expression control in retinoblastoma cells.

There were several reports about the miRNAs mentioned above. MiR-497 was already identified as a down-regulated miRNA in female breast cancer and multiple sclerosis patients $(21,22)$. Further study attributed quite characterized tumorsuppressor function to miR-497 in peritoneal carcinoma (23). MiR-491-3P was thought to play a potential role in regulating meiotic recombination- and synapsis-related genes (24). 


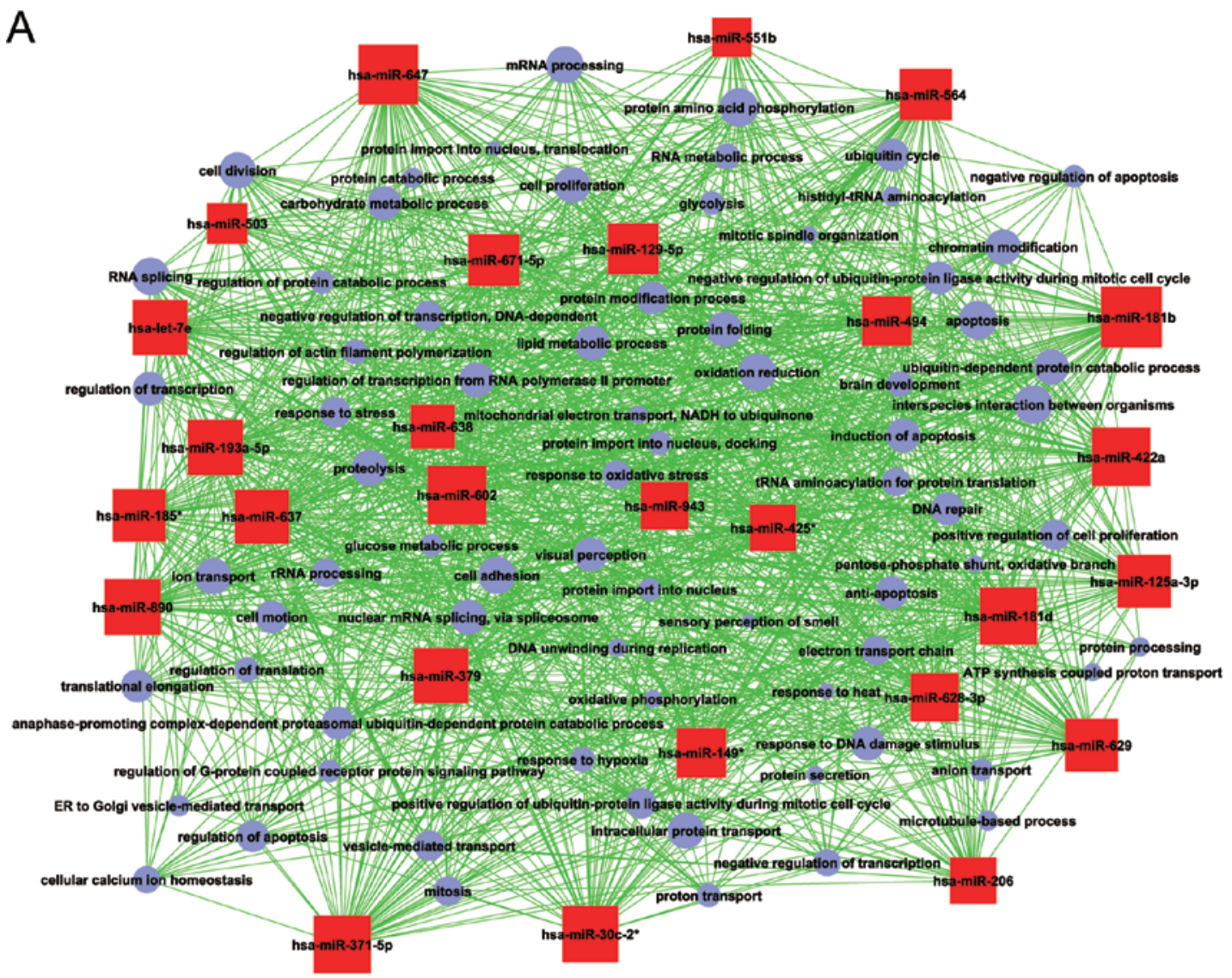

B

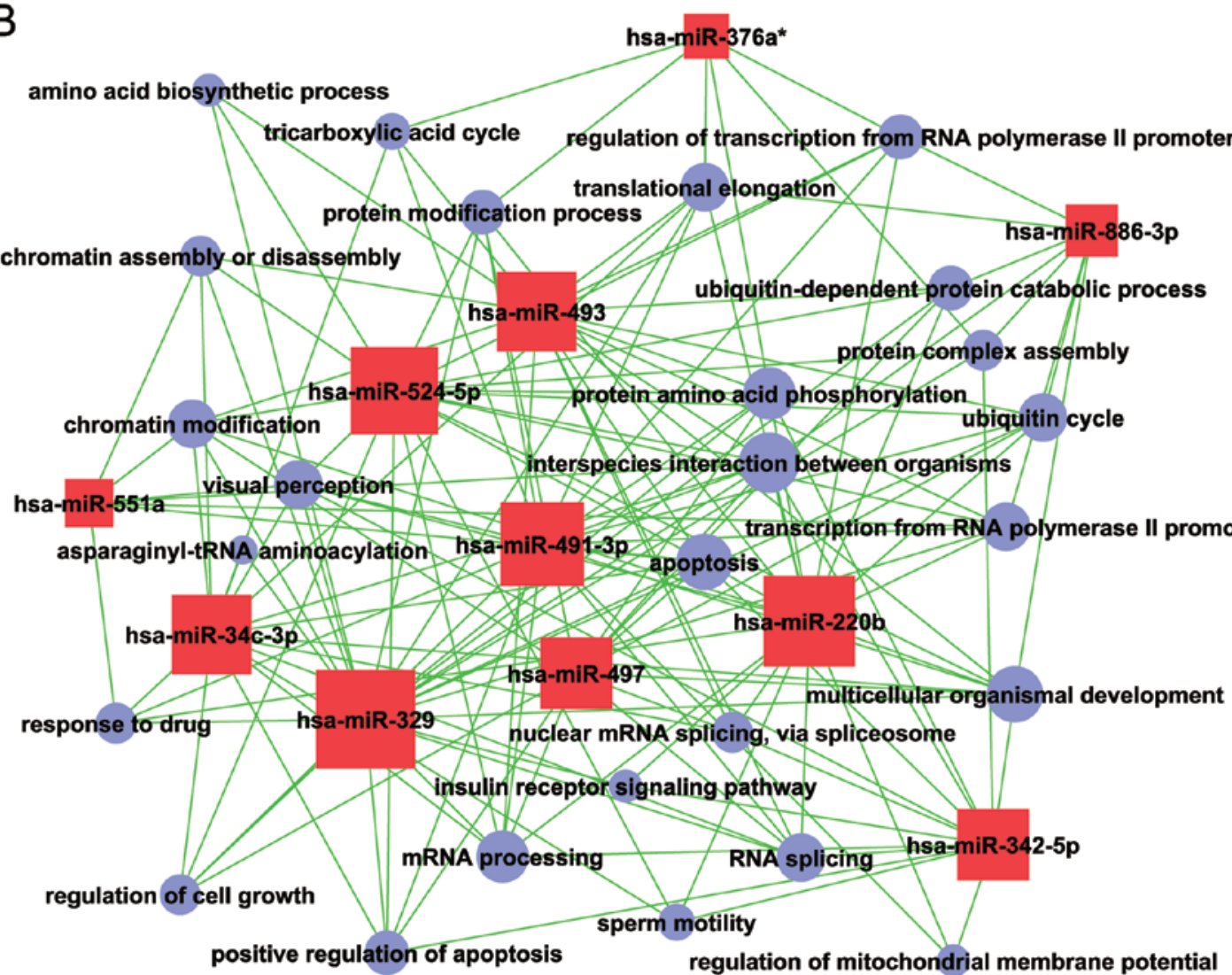

Figure 4. microRNA-Go-network. (A) shows the network of up-regulated miRNAs. The significant GO categories identified in this network are apoptosis, interspecies interaction between organisms, cell proliferation and cell adhesion with degrees of 27, 27, 26 and 25, respectively; (B) shows the network for down-regulated miRNAs. The significant GO categories in this network are interspecies interaction between organisms, apoptosis and multicellular organismal development with degrees of 10,9 and 9, respectively. Red square nodes represent miRNAs, light blue cycle nodes represent GO categories and straight lines indicate the control of miRNA on GO categories. 


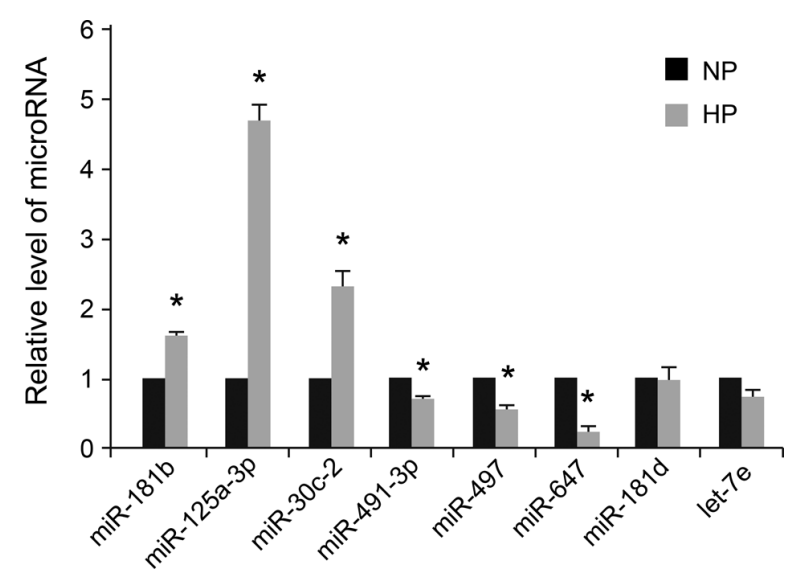

Figure 5. Validation of miRNA expression profiles by quantitative real-time RT-PCR. The levels of the miRNAs with the highest degrees were measured using real-time PCR. Triplicate assays were performed for each sample and the relative level of each miRNA was normalized to the $5 \mathrm{~S}$ rRNA. The data are presented as fold changes of miRNA levels after hypoxia, compared with normal conditions, which were set as 1 . Data are expressed as the means $\pm \mathrm{SD} .{ }^{*} \mathrm{p}<0.01$

A

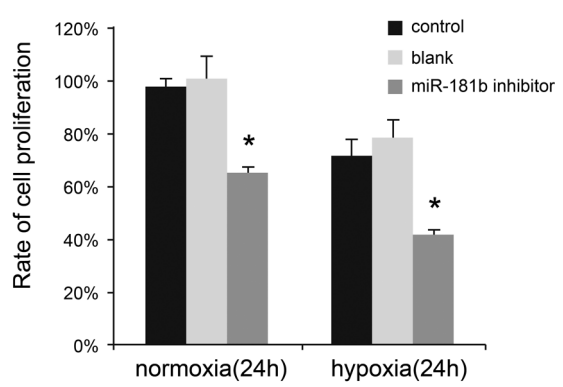

B

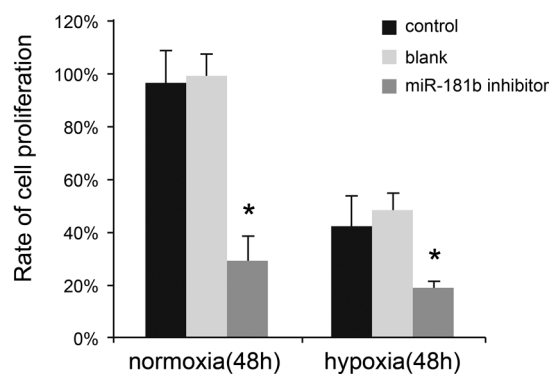

Figure 6. MiR-181b inhibitor inhibits proliferation of RB cells. Cell proliferation was measured by CCK-8. (A and B) showed RB cells proliferation rate 24 and $48 \mathrm{~h}$ after transfection respectively. Cell proliferation was suppressed by miR $181 \mathrm{~b}$ inhibitor $24 \mathrm{~h}$ after transfection, and the effects last until $48 \mathrm{~h}$, compared with normal blank, which was set as $100 \%$. "p $<0.01$.

MiR-125a-3p was down-expressed in K-ras-mutated HBECs and NSCLC cell lines and may regulate cellular proliferation, angiogenesis and inflammation (Hazra et al, Cancer Prev Res 3: abs. 31, 2010). Among the HRMs detected in our study, miR-181b was especially interesting. Not only did miR-181b display the highest degree in the network, but several studies have revealed that miR-181b has an intimate relationship with malignant tumors. Studies have demonstrated that miR-181b is highly expressed in acute lymphocytic leukemia (25) and colorectal cancer patients (26). Further, the expression level of miR-181b was found to be strongly associated with clinical response to S-1 in colon cancer patients (27). More importantly, in the cases where miR-181b has been identified as a HRM in colon cancer, breast cancer and nasopharyngeal carcinoma cells (CNE), the induction of miR-181b occurred via a HIF-dependent mechanism (18). Our study also demonstrated that miR-181b was induced by hypoxia in retinoblastoma cells, and miR-181b inhibitor suppressed the proliferation of retinoblastoma cells suggesting that miR-181b may play a positively regulated role on retinoblastoma growth under hypoxia condition. Unlike other cancers, retinoblastoma is a cancer of the nervous system that arises from neural progenitor cells. Abnormal expression of miR-181b has also been identified in the central nervous system disease schizophrenia (28), suggesting that miR-181b may be a brain-specific miRNA. These questions deserve further investigation to explore the functions and mechanisms of miR-181b in the genesis and development of retinoblastoma.

The response of cancer cells to hypoxia includes a series of physiologic processes such as apoptosis, angiogenesis, proliferation and DNA damage which influence cell growth, metastasis, invasion and the response to therapy of tumors. The microRNA-GO-Network analysis organized the genes targeted by HRMs in retinoblastoma cells into hierarchical categories based on biological process and revealed that the center GO categories were apoptosis, cell adhesion, cell proliferation and mRNA processing. These GO categories have been shown to associate closely with the response of cancer cells to hypoxia. These results suggest that the HRMs we identified are valid regulatory factors involved in hypoxia.

Our study represents the first attempt to conduct an analysis of the differential expression of miRNAs in a human retinoblastoma cell line under hypoxic conditions, to identify a cluster of specific HRMs in retinoblastoma and to explore their functions in the response of cancer cells to hypoxia. Although the concrete functions and downstream pathways affected by these HRMs require further study, we can speculate that these miRNAs may contribute to the variability in the cellular response to hypoxia. This speculation enhances our understanding of the mechanism of response to hypoxia and may provide new therapeutic targets for treatment of retinoblastoma. As gene regulators, HRMs have some distinct advantages as treatment targets compared to protein-coding genes. Since HRMs regulate expression at a post-transcriptional level, they do not require protein translation processes to function. This post-transcriptional regulation may be an advantage as translation is inhibited in response to low oxygen levels (29).

In conclusion, the HRMs identified in retinoblastoma cells in this study deserve further attention. We consider these findings quite significant, especially in the context of tumor development, metastasis and hypoxia response. We believe that these HRMs have the potential to be new targets for the treatment of retinoblastoma.

\section{Acknowledgments}

This work was supported by the Shanghai Leading Academic Discipline Project (No. S30205), the Shanghai Science and Technology Commission (No. 10JC1409100), the National Natural Science Foundation of China (Nos. 30901654 and 81001008), the National Key Program for Basic Research of 
China (No. 2010CB529902) and the Shanghai Health Bureau Youth Foundation (No. 2006y20).

\section{References}

1. Hu DN: Prevalence and mode of inheritance of major genetic eye diseases in China. J Med Genet 24: 584-588, 1987.

2. Shields CL and Shields JA: Diagnosis and management of retinoblastoma. Cancer Control 11: 317-327, 2004.

3. Gruber M and Simon MC: Hypoxia-inducible factors, hypoxia and tumor angiogenesis. Curr Opin Hematol 13: 169-174, 2006.

4. Koumenis C: ER stress, hypoxia tolerance and tumor progression. Curr Mol Med 6: 55-69, 2006.

5. Gordan JD and Simon MC: Hypoxia-inducible factors: central regulators of the tumor phenotype. Curr Opin Genet Dev 17: 71-77, 2007.

6. Wu JH, Xu P, Yi MY, Wang F, Wang XL and Huang Q: Analysis of angiogenesis associated factors of retinoblastoma cell line and tumor tissues. Zhonghua Yan Ke Za Zhi 41: 419-422, 2005.

7. Adithi M, Nalini V, Kandalam M and Krishnakumar S: Expression of matrix metalloproteinase and their inhibitors in retinoblastoma. J Pediatr Hematol Oncol 29: 399-405, 2007.

8. Boutrid H, Jockovich ME, Murray TG, Piña Y, Feuer WJ, Lampidis TJ and Cebulla CM: Targeting hypoxia, a novel treatment for advanced retinoblastoma. Invest Ophthalmol Vis Sci 49: 2799-2805, 2008.

9. Yang M, Li Y and Padgett RW: MicroRNAs: small regulators with a big impact. Cytokine Growth Factor Rev 16: 387-393, 2005.

10. Miska EA: How microRNAs control cell division, differentiation and death. Curr Opin Genet Dev 15: 563-568, 2005.

11. Zhang J, Guo H, Qian G, Ge S, Ji H, Hu X and Chen W: MiR-145, a new regulator of the DNA fragmentation factor-45 (DFF45)mediated apoptotic network. Mol Cancer 9: 211, 2010.

12. Cha HJ, Shin S, Yoo H, Lee EM, Bae S, Yang KH, Lee SJ, Park IC, Jin YW and An S: Identification of ionizing radiationresponsive microRNAs in the IM9 human B lymphoblastic cell line. Int J Oncol 34: 1661-1668, 2009.

13. Svoboda M, Izakovicova Holla L, Sefr R, Vrtkova I, Kocakova I, Tichy B and Dvorak J: Micro-RNAs miR125b and miR137 are frequently upregulated in response to capecitabine chemoradiotherapy of rectal cancer. Int J Oncol 33: 541-547, 2008.

14. Song X, Zhou Y, Jia R, Xu X, Wang H, Hu J, Ge S and Fan X: Inhibition of retinoblastoma in vitro and in vivo with conditionally replicating oncolytic adenovirus H101. Invest Ophthalmol Vis Sci 51: 2626-2635, 2010.

15. Gene Ontology Consortium: The Gene Ontology (GO) project in 2006. Nucleic Acids Res 34: D322-D326, 2006.

16. Kulshreshtha R, Ferracin M, Wojcik SE, Garzon R, Alder H, Agosto-Perez FJ, Davuluri R, Liu CG, Croce CM, Negrini M, Calin GA and Ivan M: A microRNA signature of hypoxia. Mol Cell Biol 27: 1859-1867, 2007.
17. Hebert C, Norris K, Scheper MA, Nikitakis N and Sauk JJ: High mobility group A2 is a target for miRNA-98 in head and neck squamous cell carcinoma. Mol Cancer 14:6:5, 2007.

18. Hua Z, Lv Q, Ye W, Wong CK, Cai G, Gu D, Ji Y, Zhao C, Wang J, Yang BB and Zhang Y: MiRNA-directed regulation of VEGF and other angiogenic factors under hypoxia. PLoS One 1: e116, 2006

19. Donker RB, Mouillet JF, Nelson DM and Sadovsky Y: The expression of Argonaute 2 and related microRNA biogenesis proteins in normal and hypoxic trophoblasts. Mol Hum Reprod 13: 273-279, 2007

20. Camps C, Buffa FM, Colella S, Moore J, Sotiriou C, Sheldon H, Harris AL, Gleadle JM and Ragoussis J: hsa-miR-210 is induced by hypoxia and is an independent prognostic factor in breast cancer. Clin Cancer Res 14: 1340-1348, 2008.

21. Yan LX, Huang XF, Shao Q, Huang MY, Deng L, Wu QL, Zeng YX and Shao JY: MicroRNA miR-21 overexpression in human breast cancer is associated with advanced clinical stage, lymph node metastasis and patient poor prognosis. RNA 14: 2348-2360, 2008.

22. Lindberg RL, Hoffmann F, Mehling M, Kuhle J and Kappos L: Altered expression of miR-17-5p in CD4 $4^{+}$lymphocytes of relapsingremitting multiple sclerosis patients. Eur J Immunol 40: 888-898, 2010.

23. Flavin RJ, Smyth PC, Laios A, O'Toole SA, Barrett C, Finn SP Russell S, Ring M, Denning KM, Li J, Aherne ST, Sammarae DA, Aziz NA, Alhadi A, Sheppard BL, Lao K, Sheils OM and O'Leary JJ: Potentially important microRNA cluster on chromosome $17 \mathrm{p} 13.1$ in primary peritoneal carcinoma. Mod Pathol 22: 197-205, 2009.

24. Lian J, Zhang X, Tian H, Liang N, Wang Y, Liang C, Li X and Sun F: Altered microRNA expression in patients with nonobstructive azoospermia. Report Biol Endocrinol 11: 7-13, 2009.

25. Zanette DL, Rivadavia F, Molfetta GA, Barbuzano FG, ProtoSiqueira R, Silva-Jr WA, Falcão RP and Zago MA: miRNA expression profiles in chronic lymphocytic and acute lymphocytic leukemia. Braz J Med Biol Res 40: 1435-1440, 2007.

26. Xi Y, Formentini A, Chien M, Weir DB, Russo JJ, Ju J, Kornmann $\mathrm{M}$ and $\mathrm{Ju} \mathrm{J}$ : Prognostic values of microRNAs in colorectal cancer. Biomark Insights 2: 113-121, 2006.

27. Nakajima G, Hayashi K, Xi Y, Kudo K, Uchida K, Takasaki K, Yamamoto $\mathrm{M}$ and Ju J: Non-coding microRNAs hsa-let-7g and hsa-miR-181b are associated with chemoresponse to S-1 in colon cancer. Cancer Genom Proteomics 3: 317-324, 2006

28. Beveridge NJ, Tooney PA, Carroll AP, Gardiner E, Bowden N, Scott RJ, Tran N, Dedova I and Carins MJ: Dysregulation of miRNA $181 \mathrm{~b}$ in the temporal cortex in schizophrenia. Hum Mol Genet 17: 1156-1168, 2008.

29. Kraggerud SM, Sandvik JA and Pettersen EO: Regulation of protein synthesis in human cells exposed to extreme hypoxia. Anticancer Res 15: 683-686, 1995. 